\title{
Random Noise Generation Using Fourier Series
}

\author{
Jared A. Grauer* \\ NASA Langley Research Center, Hampton, Virginia 23681
}

\section{Introduction}

Simulating the flight of an aerospace vehicle has many important applications, including rehearsing mission profiles, refining feedback control or estimation algorithms, and assessing risks in launch trajectories. These computer simulations are often run in a Monte Carlo environment, where random noise sequences are used to systematically introduce uncertainties into the model. Some examples of noise sequences include measurement noise on sensor outputs, atmospheric turbulence, model structure error from ignored physics, and drift in clock rates or calibration parameters.

Because insights gained from simulation analyses can have significant impact, it is important to use accurate tools for noise generation. As an example, sensor outputs are often modeled as having zero-mean additive measurement noise and a random bias. If the simulated measurement noise is not zero-mean, then the non-zero mean then becomes confounded with the bias, which may mislead the interpretation of results in extreme cases.

The standard method for generating random noise sequences is to query a (pseudo) random number generator algorithm, which produces a sequence from a uniform distribution. True random number generators can also be used, but are less suitable for Monte Carlo simulation due to hardware costs and latencies in generating potentially many and large sequences of random numbers [1]. The random sequence is then mapped to the desired distribution, passed through coloring or shaping filters to tailor the power spectrum (called spectral factorization), and then scaled to the desired level.

A different method for noise generation was proposed in Ref. [2], which is referred to here as the Lanczos method. After subjecting many sequences from different random number generators to Fourier analysis, the authors hypothesized that a Gaussian "ideal white noise pattern" could be synthesized, using the Fourier series, by summing harmonic sinusoids with constant amplitudes and random phase angles drawn from a uniform distribution. By extension, the spectral coloring of the noise could be designed by appropriate selection of the sinusoid amplitudes. This idea has also been mentioned by others, but is often limited to introductory descriptions, obscured in rigorous mathematical treatments, or mentioned informally in online

*Research Engineer, Dynamic Systems and Control Branch, MS 308. Senior Member AIAA. 
forums $[3-6]$.

This Note describes the Lanczos method for noise generation, as reported in Ref. [2], and presents applications for aircraft simulation. Comparisons are made with conventional methods of noise generation, and it is shown that the noise sequences generated using the Lanczos method most often have more accurate statistical characteristics than those generated using the standard methods. Although the standard methods already produce high-quality noise sequences, using the Lanczos method to generate noise, when applicable, can realize a more accurate representation of the desired noise, and therefore lead to clearer insights into simulation analyses. It is hoped that others may find this description of the noise generation procedure and these simulation examples interesting and helpful for their own uses.

\section{Method}

The strategy for constructing Gaussian noise sequences, as described in Ref. [2], is to combine many harmonic sinusoids having specified amplitudes and uniformly-distributed phase angles. To do this, define

$$
N=\frac{T}{\Delta t}+1
$$

samples at the times

$$
t_{i}=(i-1) \Delta t, \quad \text { for } i=1,2, \ldots, N
$$

where $T$ is the data record length, $\Delta t$ is the sampling period, and $i$ is the time index. The harmonic frequencies of the record are

$$
f_{k}=\frac{k}{T}, \quad \text { for } k=1,2, \ldots, N / 2
$$

where $k$ is the integer harmonic number. The noise sequence is then synthesized as the Fourier series

$$
v\left(t_{i}\right)=\frac{c_{0}}{2}+\sum_{k=1}^{N / 2} c_{k} \sin \left(2 \pi f_{k} t_{i}+\phi_{k}\right)
$$

where $c_{k}$ is the amplitude spectrum and $\phi_{k}$ is the phase spectrum. The bandwidth of the sequence is bounded by the fundamental frequency $1 / T$ and the Nyquist frequency $N / 2 T$. The harmonic sinusoid basis functions in the Fourier series are a natural choice for describing the noise because of their mutual orthogonality and because of the intuitive concepts of frequency, bandwidth, and power.

The $c_{k}$ values are selected according to the desired power spectrum. For example, white noise has equal values, or Brownian noise has values proportional to $f_{k}^{-2}$. The amplitudes could instead be chosen to match empirical data observed from experiment. Setting any values of $c_{k}$ to zero removes power from the noise at that frequency, similar to applying an ideal band-stop filter to the sequence. Whereas coefficient values 
in a coloring filter can be adjusted during the simulation, the Fourier approach generally restricts $c_{k}$ and $\phi_{k}$ values to remain constant in time over the record length. The bias term $c_{0}$ is included to offset any numerical round-off errors and yield a zero-mean sequence. The $\phi_{k}$ values are drawn randomly from a uniform distribution over the interval $[0,2 \pi)$.

\section{Examples}

This section presents three examples for generating noise sequences using the Lanczos method. The first example is a unit-variance white noise sequence to be scaled and added to sensor outputs as measurement noise. The second example is colored noise for representing unmodeled dynamics, both from a qualitative perspective and using experimental data. The last example is the simulation of atmospheric turbulence using the von Kármán model. Examples using standard methods of noise generation are also given for comparison.

\section{III.A. Measurement Noise}

White noise is a zero-mean, normally-distributed, uncorrelated random sequence with constant power over frequency. This type of noise is often applied as measurement noise to corrupt sensor outputs, or is input to a shaping filter to create colored noise. Measurement noise can be constructed using Fourier series by selecting equal values for the amplitude spectrum. A unit-variance signal can be formed by selecting $c_{k}=2 / \sqrt{N}$, or by normalizing the sequence by its sample variance [7]. The Lanczos method creates noise sequences with zero mean automatically because all harmonic sinusoids have an integer number of cycles within the record length and because $c_{0}$ removes any non-zero mean due to small numerical round-off errors.

Two realizations of a unit-variance measurement noise sequence, $10 \mathrm{~s}$ in duration and sampled at $50 \mathrm{~Hz}$ $(N=501)$, were generated and are shown in Fig. 1 . The first sequence was created using the Lanczos method. Phase angles were drawn using the rand.m function in MATLAB ${ }^{\circledR}$, which implements the Mersenne-Twister algorithm for generating random numbers. Here 250 sinusoids were combined, each with $c_{k}=0.0894$. The second sequence was generated in a more conventional manner using the randn.m function, which also uses the Mersenne-Twister algorithm and applies the Ziggurat algorithm for achieving a normal distribution. The first sequence had sample mean and variance of +0.0000 and 1.0000 , whereas the second sequence had +0.1369 and 1.0375. In terms of the desired mean and variance ( 0 and 1$)$, the sequence generated using the Lanczos method was more accurate. Although this example shows only one realization of the noise sequences, Monte Carlo analysis in Ref. [7] suggests this result (and others shown later) is representative of the methods.

As described in Refs. $[2,8]$, the phase distribution of noise is random and assumed to resemble a uniform distribution. One way to view the distribution of phase angles is the empirical cumulative distribution 


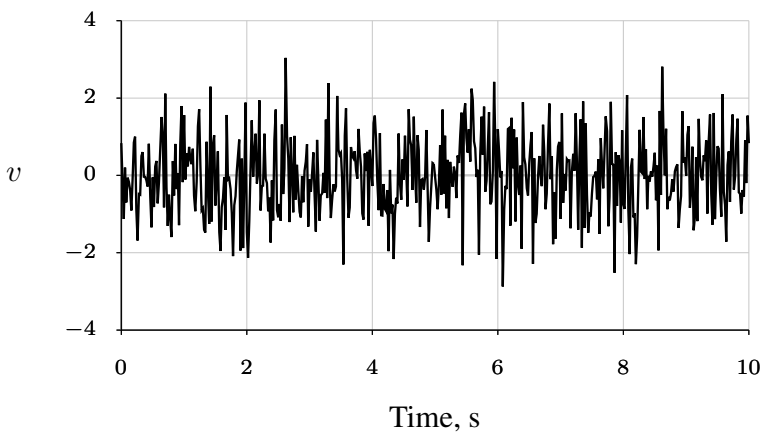

(a) Lanczos method

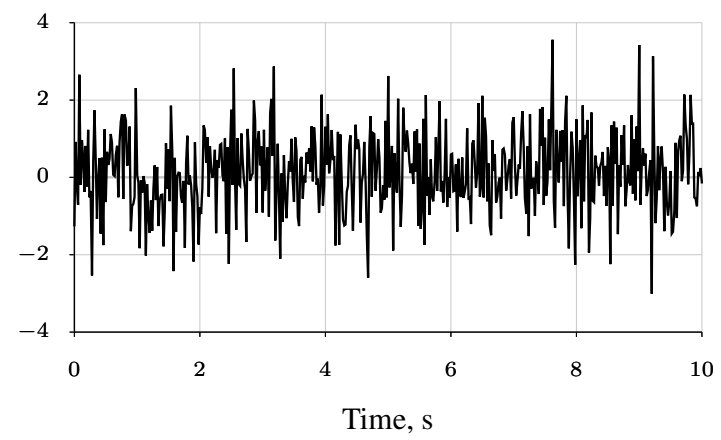

(b) Standard method

Figure 1. Measurement white noise sequences

function $(\mathrm{CDF})$. There are other methods, but these typically require analyst judgment, for example choosing bin sizes in a histogram. For a uniform distribution, the CDF is a straight line, and any large or systematic deviations from a straight line indicate a different distribution. The noise sequences in Fig. 1 were first transformed into the frequency domain using a high-accuracy Fourier transform [9]. The phase angles were then computed, sorted, and ordered to form the empirical CDFs, as shown in Fig. 2. Both sequences followed the theoretical line reasonably well, with $R^{2}$ values of 0.99 and 0.98 for sequences constructed using the Lanczos method and standard method, respectively. For the standard method, phase angles were slightly underrepresented in the first three quadrants, but for the Lanczos method, this was not the case. Overall, these phase angles represented uniform distributions well.

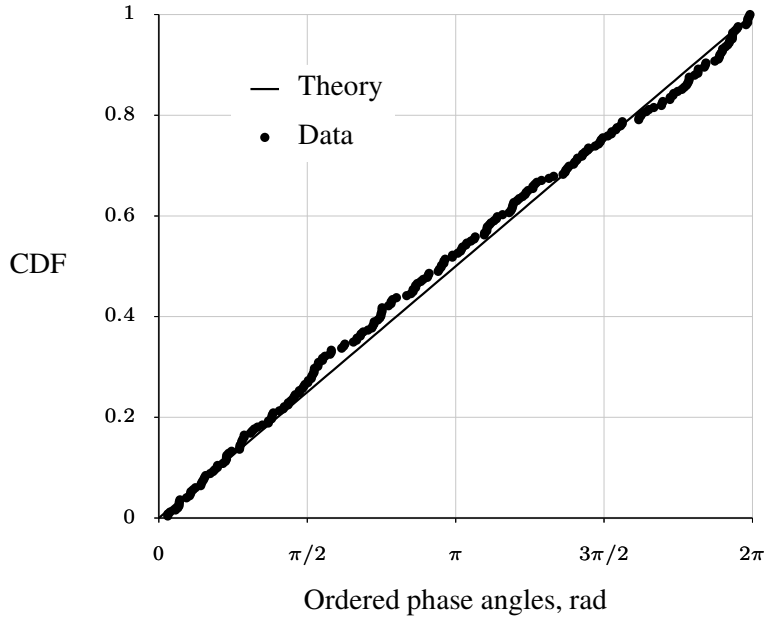

(a) Lanczos method

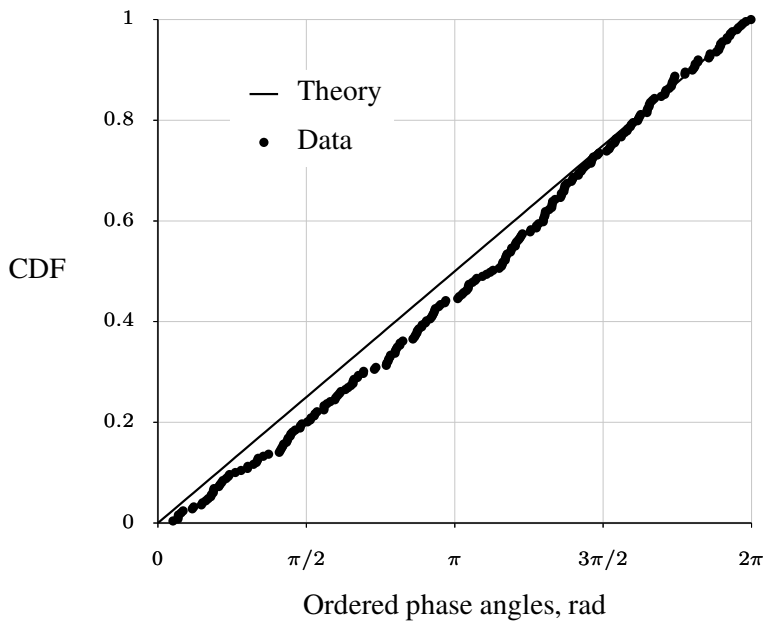

(b) Standard method

Figure 2. Cumulative distribution functions of the measurement noise phase angles

Another characteristic of white noise, the absence of serial correlations in time, can be examined using the sample autocorrelation function

$$
\mathcal{R}_{v v}(l)=\frac{1}{N} \sum_{i=1}^{N-l} v(i) v(i+l), \quad \text { for } l=0,1,2, \ldots, N-1
$$


where $l$ is the lag index. For white noise, the autocorrelation should be statistically zero at all lag values except $l=0$, where it equals the sample variance. Figure 3 shows the autocorrelation of the two measurement noise sequences from Fig. 1. The autocorrelation of the sequence constructed using the Lanczos method was 0.9980 at $l=0$, which was close to the desired value of 1 , and all other values remained within the $2 \sigma$ bound, computed from $[9]$

$$
s\left[\mathcal{R}_{v v}(l)\right]=\frac{r_{v v}(0)}{\sqrt{N}}=0.0447, \quad \text { for } l \neq 0
$$

The second sequence was 1.0542 at $l=0$, had 22 values ( $2.2 \%$ of the total samples) beyond the $2 \sigma$ bound, and in general had larger values of autocorrelation at all lag values. The sequence generated using the Lanczos method was less correlated with lagged versions of itself than that generated using the standard method, and was therefore more representative of white noise.

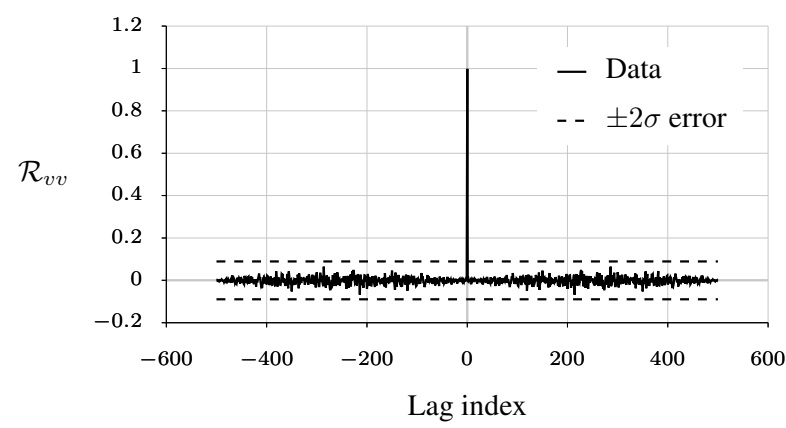

(a) Lanczos method

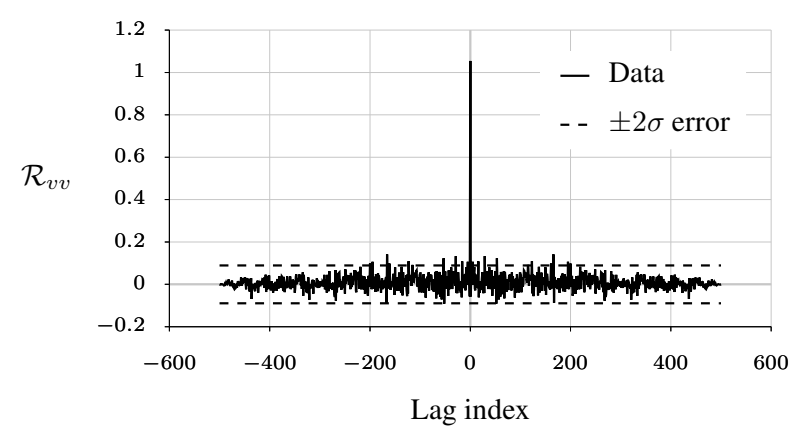

(b) Standard method

Figure 3. Autocorrelations of the measurement noise sequences

The power spectrum of white noise is constant with frequency. One way to estimate the power spectrum is with the one-sided periodogram [10]

$$
G_{v v}\left(j \omega_{k}\right)=\frac{2}{T}\left|v\left(j \omega_{k}\right)\right|^{2}
$$

where $j=\sqrt{-1}, \omega_{k}=2 \pi f_{k}$, and $v\left(j \omega_{k}\right)$ represents the Fourier transform of $v\left(t_{i}\right)$. Power spectral density estimates for the two noise sequences in Fig. 1 are shown in Fig. 4. For harmonic data having integer numbers of cycles, such as the noise sequence constructed using the Lanczos method, the periodogram is an accurate representation of the power spectrum. Equation (7) was evaluated at the harmonic frequencies $f_{k}$. Note that because the Fourier series is a collection of sinusoids at discrete frequencies, there is no power at frequencies that are non-integer multiples of $1 / T$. The power spectrum for the Fourier-based sequence, shown in Fig. 4(a), was within machine precision of the desired value of 0.04 at all frequencies, and therefore was a good representation of white noise.

For generic sequences with continuous power spectra, the periodogram method produces estimates with large errors [11]. To reduce that error for the second signal, the Fourier transform was computed at a 
frequency resolution five times finer than before, and the binning method [9] was applied. This resulted in the same frequency resolution on the power spectrum, but with five times less error than would have resulted using the normal periodogram method. The resulting spectral estimate for the second sequence, shown in Fig. 4(b), had more scatter about the desired value of 0.04 , but on average (over frequency) was as accurate as the Lanczos method. Therefore, both sequences seem to be good at achieving a flat power spectrum, considering that some additional averaging was needed for viewing the power spectrum of the second sequence. Note however that the autocorrelation and power spectral density are Fourier transform pairs and the autocorrelation of the Fourier-based sequence was more accurate, so it is likely that this sequence also has a more accurate power spectrum. This was to be expected because the Lanczos method prescribes the power spectrum for the noise, and because the method used for analysis was matched to the way that the Lanczos method generates the random sequence.

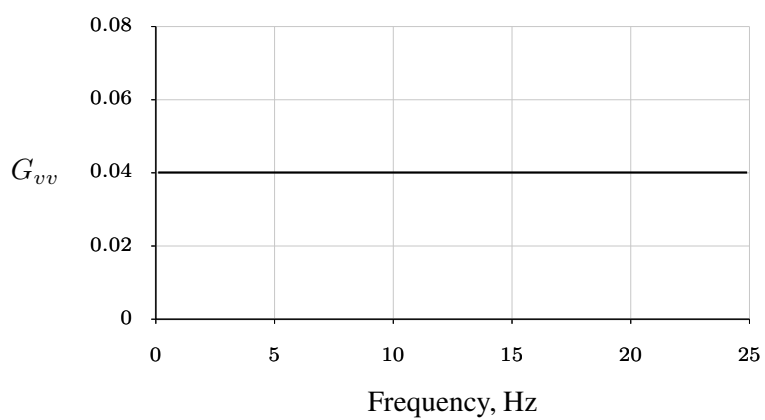

(a) Lanczos method

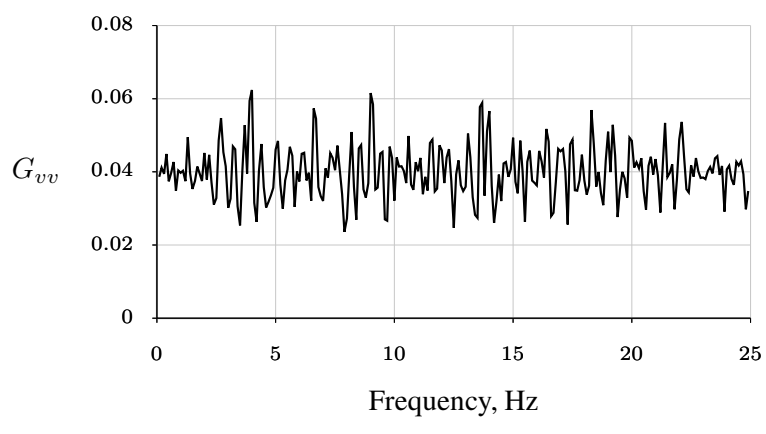

(b) Standard method

Figure 4. Power spectral density estimates of the measurement noise sequences

Whereas the Ziggurat algorithm was used to explicitly map the uniform distribution to a normal distribution for the standard method, the Lanczos method relies on the central limit theorem for justification in achieving a normal distribution - if enough sinusoids are combined, the distribution tends toward a Gaussian. Results in Ref. [7] indicated that about 50 sinusoids (or $2 \mathrm{~s}$ of data sampled at $50 \mathrm{~Hz}$ ) were needed on average to achieve a normal distribution within $1 \%$ error, which seems relatively efficient. To check the normality of the two noise sequences, probability plots [12] were constructed. Given a desired distribution, probability plots map sequences to straight lines, so that any deviation from the line, and therefore from the distribution, can be visualized more easily. Figure 5 shows this transformation for the noise sequences in Fig. 1. Except for small variations in the standard method, both plots follow the lines relatively well, with $R^{2}$ values of 0.9980 and 0.9779 for the Lanczos method and standard method, respectively. The small amounts of variation near the ends are typical of distributions with tails that decrease to zero [12]. Therefore, both sequences were described well by a normal distribution.

In this example, two measurement noise sequences, modeled as unit-variance white noise, were compared. Both the sequence constructed using the Fourier series and the sequence generated from a conventional 


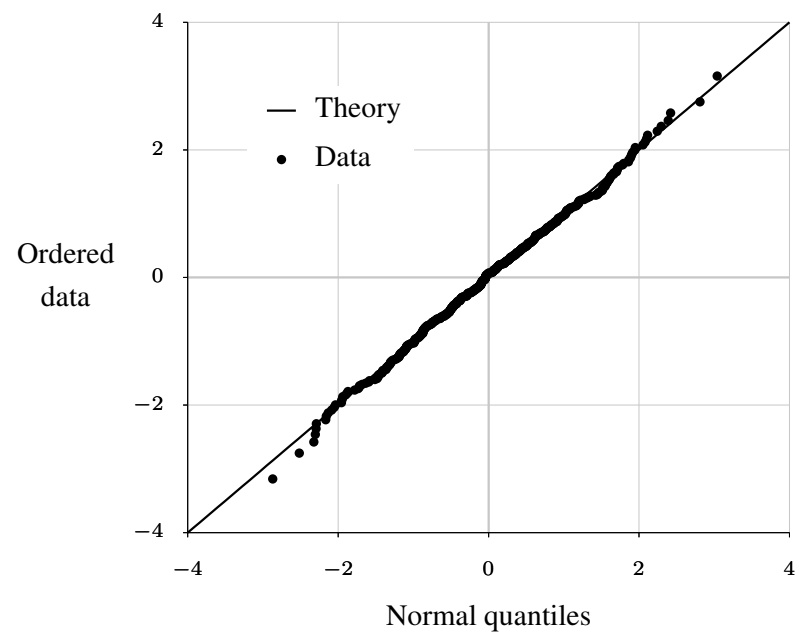

(a) Lanczos method

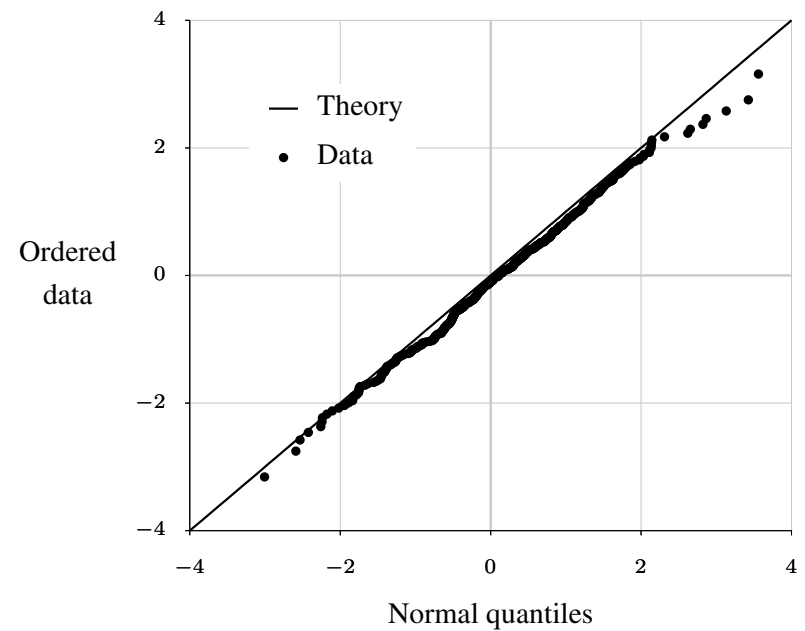

(b) Standard method

Figure 5. Normal probability plots of the measurement noise sequences

method were of good quality. However, the Fourier-based method produced sequences with more accurate sample means, variances, and autocorrelations. A direct comparison of the power spectra of the signals was not possible, but it appears both were good representations of white noise in this regard, as well as with respect to the normality of the sequences.

\section{III.B. Unmodeled Dynamics}

Discrepancies between simulation data and flight test data usually contain some deterministic content from unmodeled physics. Examples include cross-axis coupling, structural or slosh dynamics, and unsteady aerodynamics. These effects can be represented by adding colored noise to the simulation outputs, which was done in Refs. $[13,14]$ by filtering white noise with a fifth-order, low-pass, Chebyshev filter which passed frequencies near the rigid-body modes. Unmodeled dynamics can also be simulated using Fourier series by combining multiple white noise sequences having different amplitudes and bandwidths to mimic different physical phenomena.

A colored noise example, used to simulate measurements from an angle of attack vane on a subscale airplane [15], is shown in Fig. 6 with $T=10 \mathrm{~s}$ and $N=501$. The power spectrum of the noise is shown in Fig. 6(a), where the different lines indicate different physical sources. Content from 0-2 $\mathrm{Hz}$ represents unmodeled low-frequency dynamics near the rigid-body modes, power at $7 \mathrm{~Hz}$ represents the first bending mode of an air-data boom, and wide-band content up to the $25 \mathrm{~Hz}$ Nyquist frequency represents measurement noise. These components have amplitudes corresponding to $14 \%, 6 \%$, and $7 \%$ of the variation in the angle of attack time history, respectively. Noise levels and bandwidths can be selected from intuition and past experience, curve fit from experimental data, taken from manufacturer data sheets, or determined using first principles. In Fig. 6(b), the response in angle of attack $\alpha$ to a multistep elevator input is shown, as well as 
the total simulated measurement $\alpha_{m}$ and the generated noise sequence $v$.

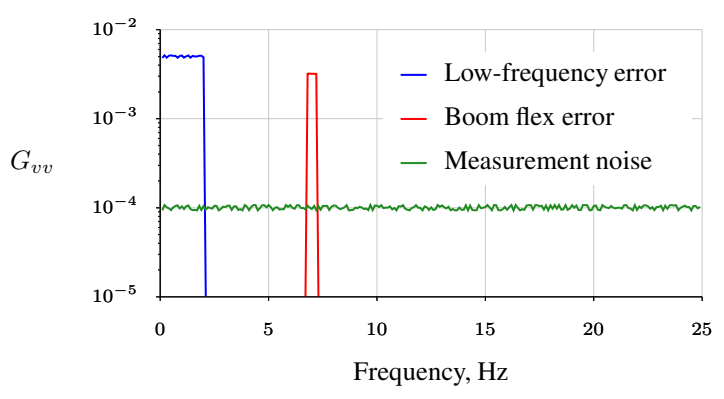

(a) Power spectrum
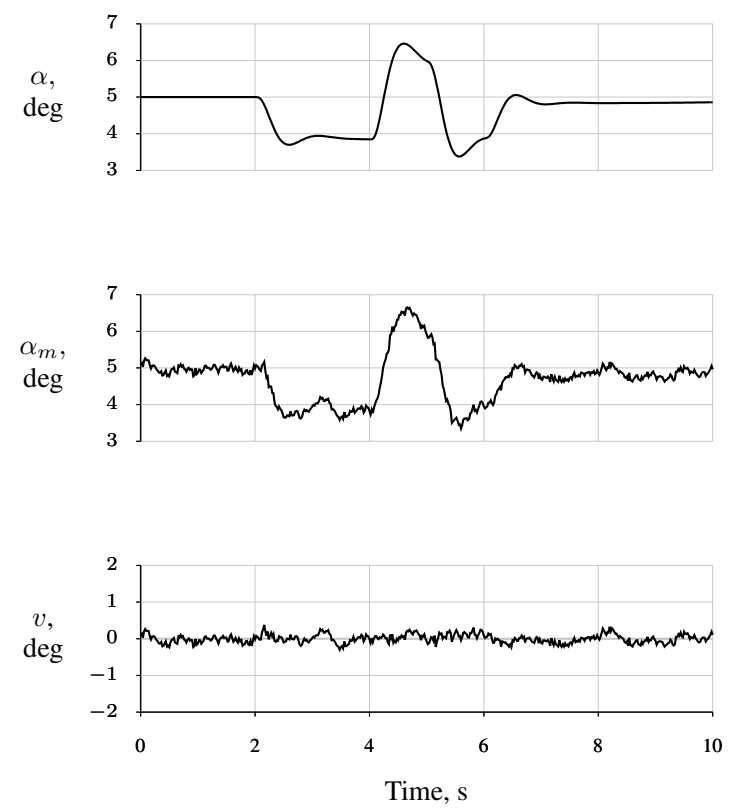

(b) Time history

Figure 6. Simulated colored noise on an angle of attack vane measurement

Alternatively, realistic noise sequences can be realized by analogy with experimental modeling data. Figure 7(a) shows angle of attack measurements from a longitudinal maneuver of the F-18 High Alpha Research Vehicle (HARV), included with Ref. [16], where $T=14 \mathrm{~s}$ and $N=701$. The modeling residual $\nu$ for the angle of attack resulting from an output-error analysis [9] is shown in Fig. 7(b) as a time history and in Fig. 7(c) as a power spectral density. The spectrum illustrates the noise floor, power in the low frequencies near the rigid-body modes, and power between $6-14 \mathrm{~Hz}$ due to structural bending in the airdata boom. By decomposing this modeling residual into a Fourier series matching the component amplitudes, randomizing the phase angles, and then recombining the sinusoids, many different and authentic realizations of this colored noise sequence can be generated. One realization is shown in Fig. 8, which looks similar in character to the original sequence in Fig. 7(b). The power spectrum of this realization, shown in Fig. 8(b), is practically identical to the experimental data in Fig. 7(c) used for its generation, as expected. 


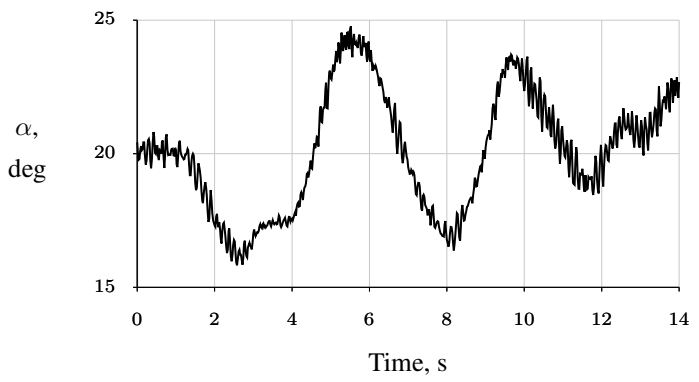

(a) Angle of attack measurements

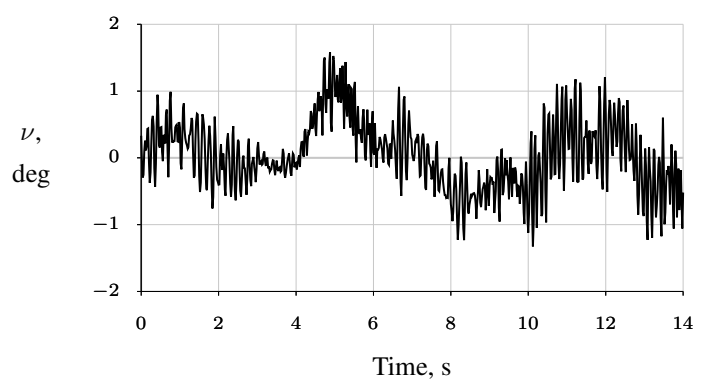

(b) Angle of attack modeling residual

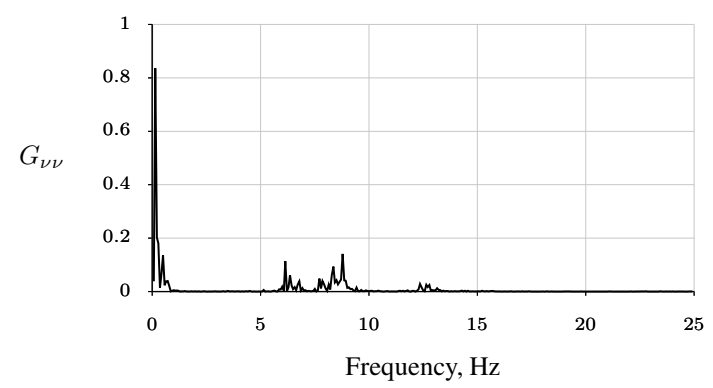

(c) Power spectrum of residual

Figure 7. HARV flight test and modeling data

\section{9 of 13}




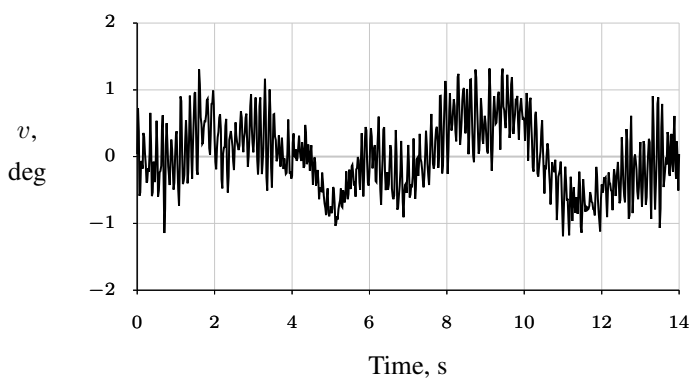

(a) Colored noise time history

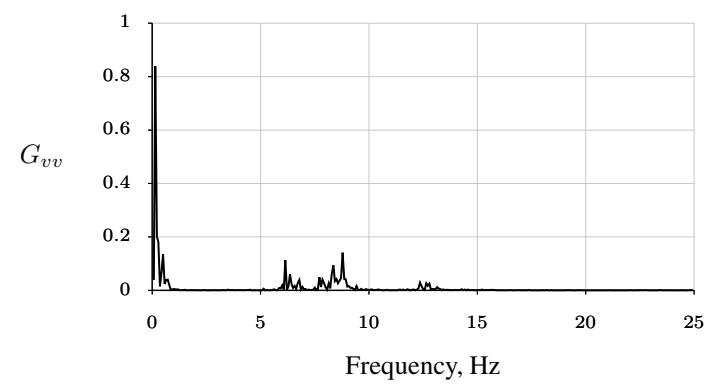

(b) Power spectrum of residual

Figure 8. A realization of colored noise generated from HARV modeling data

\section{III.C. Atmospheric Turbulence}

Military specifications prescribe power spectra of atmospheric turbulence models for flight dynamics simulations and recommend using the von Kármán model for best realism. However, this model includes irrational polynomials that can not be directly factored into coloring filters for spectral factorization. Consequently, the Dryden model is often used, although it is less representative of observed turbulence data. Other approaches for using the von Kármán model include curve fitting the power spectrum [17], discretizing the power spectra using a small number of sinusoids [18], and using Fourier transforms to apply the irrational coloring filters in the frequency domain [19].

The power spectrum of the von Kármán model for the vertical gust velocity $w$ is [20]

$$
\Phi_{w w}(j \omega)=\frac{\sigma^{2} L}{\pi V} \frac{1+\frac{8}{3}(1.339 L \omega / V)^{2}}{\left[1+(1.339 L \omega / V)^{2}\right]^{11 / 6}}
$$

where $V$ is the trim airspeed, $\sigma$ is the turbulence intensity, and $L=2500 \mathrm{ft}$ for the turbulence length scale at medium/high altitudes. The Lanczos method can be used to create these gust sequences by selecting the sinusoid amplitudes as

$$
c_{k}=\sqrt{2 \Delta \omega \Phi_{w w}\left(j \omega_{k}\right)}
$$

where $\Delta \omega=2 \pi / T$ is the angular frequency resolution of the Fourier series and where $\Phi_{w w}\left(j \omega_{k}\right)$ are evaluations of Eq. (8) at the harmonic frequencies. For comparison with spectral factorization, the coloring filter 
having the frequency response [17]

$$
H_{w}(j \omega)=\sigma \sqrt{\frac{L}{\pi V}} \frac{1+2.7478\left(\frac{L}{V}\right) j \omega-0.3398\left(\frac{L}{V}\right)^{2} \omega^{2}}{1+2.9958\left(\frac{L}{V}\right) j \omega-1.9754\left(\frac{L}{V}\right)^{2} \omega^{2}-0.1539\left(\frac{L}{V}\right)^{3} j \omega^{3}}
$$

was used, where $s$ is the Laplace variable. Equation (10) is based on a curve-fitted approximation to the von Kármán power spectrum using rational polynomials.

Two turbulence sequences were generated using the Lanczos method and spectral factorization, and are shown in Fig. 9. The data record length was selected as $T=500 \mathrm{~s}(N=25001)$ in order to examine low frequencies below the "knee in the curve;" however, only $100 \mathrm{~s}$ of the sequences are shown here for clarity. The airspeed and altitude for defining the turbulence power spectra were $V=130 \mathrm{ft} / \mathrm{s}$ and $2000 \mathrm{ft}$, respectively, which could correspond to the flight of a subscale aircraft [15]. The two sequences look qualitatively similar to each other. The estimated power spectral densities are shown in Fig. 10. The periodogram shows the Fourier-based sequence has power exactly on the model spectrum. The binning method was again applied to the sequence generated using spectral factorization to reduce error, and these results generally follow the model spectrum, but not as clearly as with the Lanczos method and the periodogram. These results may look different for other methods of spectral estimation, for example using windowing and averaging techniques [10].

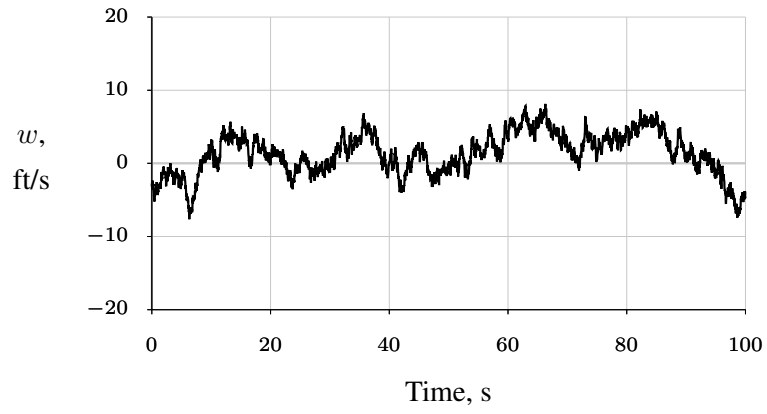

(a) Lanczos method

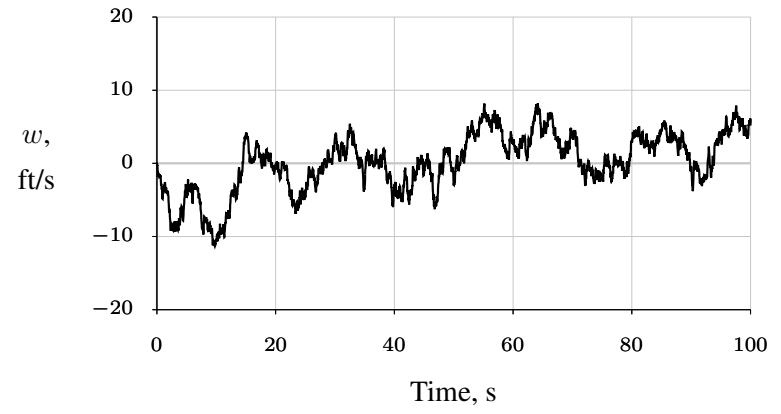

(b) Spectral factorization

Figure 9. Turbulence sequences (100 s shown of the simulated $500 \mathrm{~s}$ )

\section{Conclusions}

A simple method for generating Gaussian noise sequences with arbitrary power spectra was discussed. The method uses the Fourier series, where the amplitude spectrum is assigned according to the desired power spectra, and phase angles are drawn randomly from a uniform distribution.

The method was demonstrated using three examples. White noise was used to mimic measurement noise on simulation outputs and was shown to have accurate mean, standard deviation, autocorrelation, and power spectra, and was representative of a normal distribution. Colored noise was used to simulate 


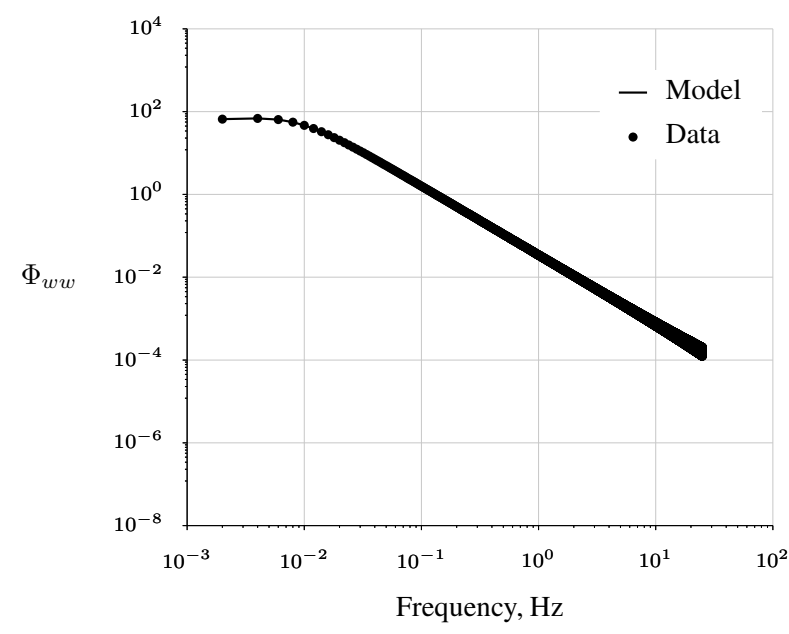

(a) Lanczos method

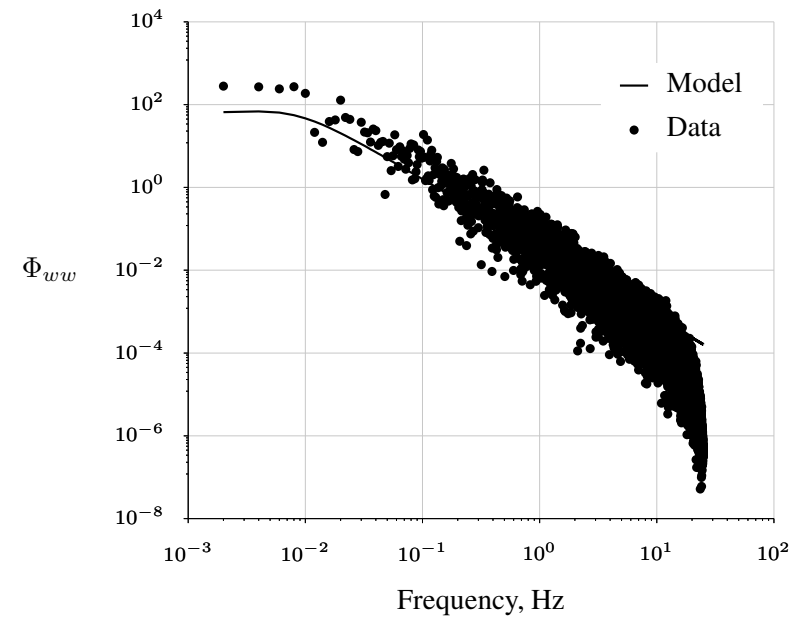

(b) Spectral factorization

Figure 10. Power spectral densities of the turbulence sequences

wide-band measurement noise, unmodeled low-frequency dynamics, air-data boom flex mode dynamics, and experimental data. Colored noise, with power spectra defined by the von Kármán model, was also used to simulate atmospheric turbulence.

The advantage of the proposed method is that random processes having arbitrary power spectra can be generated accurately, as viewed in both the time and frequency domains. The resulting noise sequences are more precisely realized and therefore are of higher quality than noise sequences constructed with traditional methods using spectral factorization. Generating random noise sequences in this way can lead to fewer Monte Carlo simulations, more realistic simulation, and less uncertainty in evaluating results and making decisions on mission risk.

\section{Acknowledgments}

This work was funded by the NASA Aeronautics Research Mission Directorate (ARMD) Learn-To-Fly project. Discussions with Eugene Morelli and members of the Dynamic Systems and Control Branch and the Flight Dynamics Branch are acknowledged and appreciated. 


\section{References}

${ }^{1}$ Haahr, M., "Introduction to Randomness and Random Numbers," http://random.org, Accessed: 2017-12-01.

${ }^{2}$ Lánczos, C. and Gellai, B., "Fourier Analysis of Random Sequences," Computers 83 Mathematics with applications, Vol. 1, No. 3-4, 1975, pp. 269-276.

${ }^{3}$ Hoblit, F., Gust Loads on Aircraft: Concepts and Applications, AIAA Education Series, AIAA, 1988, pp. $21-67$.

${ }^{4}$ Figwer, J. and Niederlizski, A., "On the Generation of High Quality White Noise Series," Applied Stochastic Models and Data Analysis, Vol. 8, No. 4, 1992, pp. 311-326.

${ }^{5}$ Figwer, J., "A New Method of Random Time-Series Simulation," Simulation Practice and Theory, Vol. 5, No. 3, March 1997, pp. 217-234.

${ }^{6}$ Giuclea, M., Mitu, A.-M., and Solomon, O., "Generation of Stationary Gaussian Time Series Compatible with Given Power Spectral Density," Proceedings of the Romanian Academy, Vol. 15, No. 3, 2014, pp. 292-299.

${ }^{7}$ Grauer, J., "A Comparison of Three Random Number Generators for Aircraft Dynamic Modeling Applications," Tech. Rep. TM-2017-219612, NASA, Hampton, VA, May 2017.

${ }^{8}$ Lánczos, C., Applied Analysis, Prentice Hall, Englewood Cliffs, NJ, 1956.

${ }^{9}$ Morelli, E. and Klein, V., Aircraft System Identification: Theory and Practice, Sunflyte Enterprises, 2016.

${ }^{10}$ Bendat, J. and Piersol, A., Random Data: Analysis and Measurement Procedures, Wiley, 4th ed., 2010.

${ }^{11}$ Press, W., Flannery, B., Teukolsky, S., and Vetterling, W., Numerical Recipes: The Art of Scientific Computing, Cambridge University Press, 1986, pp. 420-423.

${ }^{12}$ Chambers, J., Cleveland, W., Kleiner, B., and Tukey, P., Graphical Methods for Data Analysis, Statistics / Probability, Wadsworth \& Brooks/Cole, 1983.

${ }^{13}$ Maine, R. and Iliff, K., "Use of Cramer-Rao Bounds on Flight Data with Colored Residuals," Journal of Guidance and Control, Vol. 4, No. 2, March-April 1981, pp. 207-213.

${ }^{14}$ Morelli, E. and Klein, V., "Accuracy of Aerodynamic Model Parameters Estimated from Flight Test Data," Journal of Guidance, Control, and Dynamics, Vol. 20, No. 1, January-February 1997, pp. 74-80.

${ }^{15}$ Morelli, E., "GTM Polysim — Nonlinear GTM Aircraft Polynomial Simulation in MATLAB, Version 2.0," NASA Software Catalog, 2007, Number LAR-17595-1.

${ }^{16}$ Morelli, E., "System IDentification Programs for AirCraft (SIDPAC)," http://software.nasa.gov, Accessed: 2017-1201.

${ }^{17}$ Gage, S., "Creating a Unified Graphical Wind Turbulence Model from Multiple Specificiations," No. 2003-5529 in Modeling and Simulation Technologies Conference and Exhibit, AIAA, Austin, TX, August 2003.

${ }^{18}$ Beal, T., "Digital Simulation of Atmospheric Turbulence for Dryden and von Kármán Models," Journal of Guidance, Control, and Dynamics, Vol. 16, No. 1, January-February 1993, pp. 132-138.

${ }^{19}$ Campbell, C., "Monte Carlo Turbulence Simulation Using Rational Approximations to von Kármán Spectra," AIAA Journal, Vol. 24, No. 1, January 1986, pp. 62-66.

${ }^{20}$ Anon., "Flying Qualities of Piloted Airplanes," Tech. Rep. MIL-F-8785C, US Air Force, November 1980. 